\title{
EEG Patterns Prior to Motor Activations of Parasomnias: A Systematic Review
}

\author{
Milena Camaioni ${ }^{\prime}$ \\ Serena Scarpelli $\mathbb{B}^{\prime}$ \\ Maurizio Gorgoni' \\ Valentina Alfonsi $\mathbb{1 D}^{2}$ \\ Luigi De Gennaro $\mathbb{D}^{1,2}$ \\ 'Department of Psychology, Sapienza \\ University of Rome, Rome, Italy; ${ }^{2}$ IRCCS \\ Fondazione Santa Lucia, Rome, Italy
}

Introduction: Non-rapid eye movement (NREM) parasomnias are defined as abnormal nocturnal behaviors that typically arise from the NREM sleep stage 3 during the first sleep cycle. The polysomnographic studies showed an increase in sleep fragmentation and an atypical slow wave activity (SWA) in participants with NREM parasomnias compared to healthy controls. To date, the pathophysiology of NREM parasomnias is still poorly understood. The recent investigation of the EEG patterns immediately before parasomnia events could shed light on the motor activations' processes. This systematic review aims to summarize empirical evidence about these studies and provide an overview of the methodological issues.

Methods: A systematic literature search was carried out in PubMed, Web of Science, and Scopus, following the Preferred Reporting Items for Systematic Reviews and Meta-Analyses (PRISMA). The documents obtained were evaluated using the Newcastle-Ottawa Scale (NOS).

Results: Nine studies were included in the qualitative synthesis. The major evidence revealed an increased slow frequency EEG activity immediately before the motor activations in frontal and central areas and increased beta activity in the anterior cingulate cortices.

Discussion: The investigation of EEG patterns before parasomniac episodes could provide new insight into the study of NREM parasomnia pathophysiology. The high- and lowfrequency EEG increase before the episodes could represent a predictive electrophysiological pattern of the motor activations' onset. Overall, identifying specific sleep markers before parasomnias might also help differentiate between NREM parasomnias and other motor sleep disorders. Different methodological protocols should be integrated for overcoming the lack of consistent empirical findings. Thus, future studies should focus on the topographical examination of canonical EEG frequency bands to better understand spatial and time dynamics before the episodes and identify the networks underlying the onset of activations. Keywords: NREM parasomnias, episodes, motor activations, sleep EEG, polysomnography, electroencephalography

\section{Introduction}

Parasomnias are sleep disorders defined as abnormal behavior or experiential and physiological events, which can occur during specific sleep stages or sleep-wake transitions. ${ }^{1}$ The International Classification of Sleep Disorders (ICSD-III, 3rd ed) classified the parasomnias into three clusters: non-rapid eye movement (NREM) related, rapid-eye movement (REM) related, and others. ${ }^{2,3}$

Specifically, the NREM parasomnias include the sleep-related eating disorder (SRED) and the disorders of arousal (DOAs), distinguished in sleepwalking (or somnambulism), sleep terrors, and confusional arousals. REM parasomnias include
Correspondence: Luigi De Gennaro University of Rome, Via dei Marsi, 78 Rome, 00185, Italy

Tel +39-06-49917647

Fax +39-06-4991771I

Email luigi.degennaro@uniromal.it 
REM behavior disorder (RBD), recurrent isolated sleep paralysis, and nightmare disorder. Lastly, the category "other parasomnias" includes exploding head syndrome, sleep-related hallucinations, and sleep enuresis.

To date, while RBD is largely studied, ${ }^{4-6}$ evidence about NREM parasomnias is still limited. In particular, the polysomnographic (PSG) investigation has focused mainly on DOAs, especially on sleepwalking and sleep terrors. $^{7}$

NREM parasomnias are characterized by incomplete awakening episodes, impaired responsiveness to external stimuli, and partial or complete amnesia for the episode. ${ }^{1,3,8}$

These sleep disorders usually arise from slow wave sleep (SWS), mainly during the first third of the night. ${ }^{6,9-11}$ However, NREM parasomnias can also emerge during NREM stage $2 .^{6,10-12}$

The present literature reports that the macrostructure of participants with DOAs is typically preserved compared to that of a healthy control group. ${ }^{13-16}$ However, studies on sleepwalking have shown an increase in SWS fragmentation and a higher number of arousals or awakenings from SWS than a control group. ${ }^{16-19}$ The whole-night studies on patients with sleepwalking have found decreased slow wave activity (SWA) on central areas during the first cycle. $^{15,20-22}$ Sleepwalkers also show a slower SWA decay during the night than healthy controls. ${ }^{20}$

In this vein, some authors have observed that microstructural parameters are altered in DOAs. ${ }^{23}$ The cyclic alternating pattern (CAP) analysis, a measure that evaluates the EEG oscillations of arousal level during NREM sleep stages, ${ }^{11,24}$ has shown instability of NREM sleep in patients with sleepwalking, indicated by a higher CAP rate compared to healthy participants. ${ }^{11,21}$ Therefore, the studies using CAP suggest a reduced ability to sustain deep sleep in DOAs. ${ }^{13,20,25,26}$

Overall, empirical evidence regarding the mechanisms underlying the activation of NREM parasomnias is scarce. Interestingly, some studies have observed the so-called "hypersynchronous activity" (HSD, ie, continuous highvoltage delta waves $\geq 150 \mu \mathrm{V}$ ) in the sleep electroencephalogram (EEG) immediately before sleepwalking episodes, sleep terrors, or confusional arousals. ${ }^{9,27-30}$ In this regard, some authors have tried to identify specific sleep EEG markers of somnambulistic events..$^{9,14,27,31}$ However, these studies have reported controversial findings: ${ }^{32}$ HSD may occasionally, ${ }^{10,33}$ often, ${ }^{16}$ or always ${ }^{20,34}$ be associated with sleepwalking or sleep terrors. ${ }^{35}$ HSD has also been observed in sleepwalking without behavioral episodes, $, 9,14,25,35$ in healthy subjects, especially after sleep deprivation, ${ }^{10,14,35}$ and in adults with sleep apnea or periodic leg movements. ${ }^{32}$

Moreover, the stereo-electroencephalography (S-EEG) studies also provide interesting information regarding the pathophysiological substrate of NREM parasomnias, recording episodes in vivo. Specifically, a dissociated sleep-wake state between cortical and subcortical regions has been shown. ${ }^{36}$

In the last 20 years, some investigations of the EEG patterns immediately before the behavioral episodes of NREM parasomnias (BEp) have been carried out, aiming to solve the early conflicting findings and enhance knowledge concerning the episodes' onset mechanism.

Therefore, this systematic review aims to critically summarize these studies, looking for specific and predictive EEG patterns of BEp. Moreover, the second aim is to provide an overview of methodological issues to propose new insights for future research directions.

\section{Method}

\section{Research Strategy}

The systematic review was conducted following the Preferred Reporting Items for Systematic Reviews and Meta-Analyses (PRISMA). ${ }^{37}$ A systematic literature search was carried out in PubMed, Web of Science, and Scopus, for the "English language" and "journal articles," within their complete timespans until October 2020. The following keywords were used: (sleepwalking or somnambulism or sleep terrors or confusional arousals or NREM parasomnia*) AND (EEG or electroencephalogram or electroencephalography or PSG or polysomnography) AND (episode* or event* or arousal* or activation*). The search was limited to title and abstract. The results were imported into Mendeley, and duplicates were automatically removed. ${ }^{38}$ The remaining documents were manually selected according to the eligibility criteria.

\section{Study Eligibility}

Two researchers screened the articles according to the inclusion and exclusion criteria established a priori. Figure 1 shows the flow diagram that describes the steps of the systematic review. First, the researchers manually removed any duplicates missed. Then, the researchers performed a screening for the title and abstract, applying the following inclusion criteria: 1) publications in a peer-reviewed 


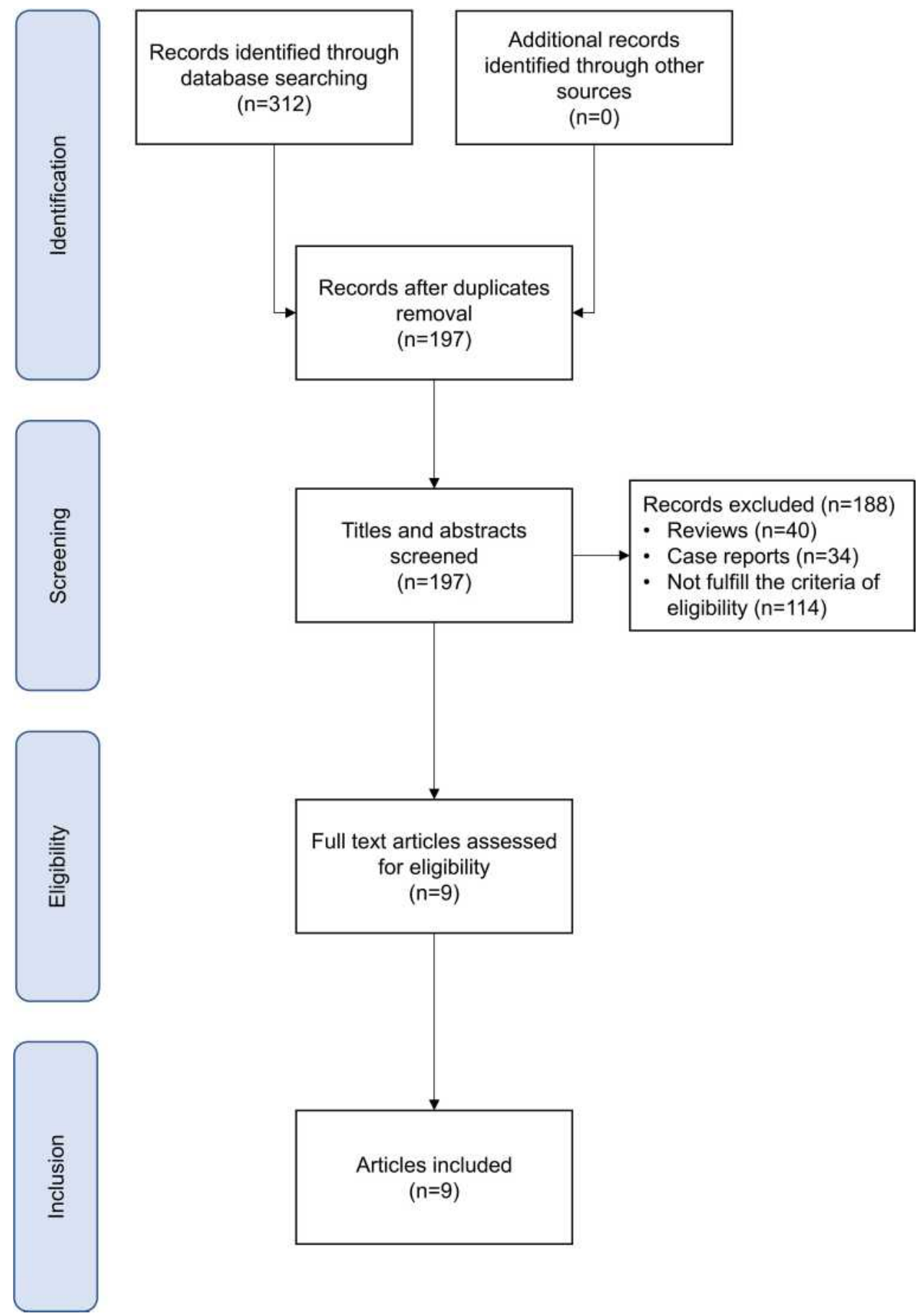

Figure I Flow diagram.

journal, 2) full-text availability, 3) articles focused on NREM parasomnia populations and 4) studies that investigated the EEG patterns immediately preceding BEp, using PSG. The exclusion criteria were: 1) review, meta-analysis, and case reports and 2) studies that did not focus on the EEG patterns prior to BEp. Finally, the methods and analysis of full-text articles obtained were screened further. The ultimate selection included solely the articles reporting established time windows before the BEp.

The participants included in the studies had to present BEp. On the other hand, we excluded those studies that included patients with neurological or psychiatric 
disorders, medical conditions, or other sleep disorders. Lastly, the references of the selected articles were also examined to retrieve documents missed by the literature search.

\section{Data Extraction}

Two researchers extracted data, developing coding sheets in Microsoft Excel. The extraction included all information related to the study of sleep EEG patterns before the BEp. First, the authors and publication year of the studies were reported. The following data were then extracted for the parasomniac group: the number of participants, ages, gender, and types of parasomnia studied. Furthermore, researchers reported personal data of eventual control groups included in the studies. Finally, the researchers extracted the information concerning BEp, night protocols, sleep EEG data, time windows before the BEp, and main results. Where possible, the examination of arousals not associated with the BEp was included.

\section{Assessment of Risk of Bias}

Two researchers evaluated the quality of the documents using the Newcastle-Ottawa Scale (NOS) for nonrandomized studies. ${ }^{39}$ The case-control studies subscale was used for assessing the risk of bias. NOS provides three domains: 1) selection, 2) comparability and 3) exposure. The highest score is 9 . A score from 9 to 7 indicates high quality, from 6 to 4 moderate quality, and from 3 to 0 low quality.

\section{Results}

\section{Search Results and Assessment of Risk of Bias}

The literature search in the database identified a total of 312 documents. After the automatic removal of duplicates, 197 papers were obtained. Overall, 188 articles were excluded. Specifically, 40 papers were reviews, and 34 were case reports, while 114 did not fulfill the eligibility criteria. A total of nine full-text articles were analyzed and included in the qualitative synthesis (see Figure 1).

The mean score of quality assessment of the nine studies was 4.67 , indicating a moderate quality Table 1 reports the score assigned to each article.

\section{Clinical and Video-Polysomnographic} Characteristics of the Included Studies

Overall, the selected studies measured the EEG patterns preceding the BEp in sleepwalking or sleep terrors. Adults
Table I Quality Assessment of Documents (Newcastle-Ottawa Quality Assessment Scale Case-Control Studies)

\begin{tabular}{|l|c|c|c|}
\hline Ref & $\begin{array}{c}\text { Selection } \\
\text { (Maximum } \\
\text { 4 Stars) }\end{array}$ & $\begin{array}{c}\text { Comparability } \\
\text { (Maximum 2 } \\
\text { Stars) }\end{array}$ & $\begin{array}{c}\text { Exposure } \\
\text { (Maximum } \\
\text { 3 Stars) }\end{array}$ \\
\hline Schenck et al ${ }^{40}$ & $\star \star$ & $\star$ & $\star \star$ \\
\hline Zadra et al ${ }^{41}$ & $\star \star$ & $\star$ & $\star \star$ \\
\hline Espa et al $^{20}$ & $\star \star \star \star$ & $\star$ & $\star \star$ \\
\hline $\begin{array}{l}\text { Guilleminault } \\
\text { et al }\end{array}$ & $\star \star$ & $\star$ & $\star \star$ \\
\hline Pilon et al & & $\star \star$ & $\star \star$ \\
\hline Jaar et al ${ }^{42}$ & $\star \star$ & & $\star \star$ \\
\hline Perrault et al & $\star \star$ & & $\star \star$ \\
\hline $\begin{array}{l}\text { Januszko } \\
\text { et al }{ }^{44}\end{array}$ & $\star \star$ & & \\
\hline $\begin{array}{l}\text { Desjardins } \\
\text { et al }{ }^{45}\end{array}$ & $\star \star$ & & \\
\hline
\end{tabular}

or young adults with an age range between 18 and 38 years were studied. Table 2 details the types of parasomnia. Five studies included participants with sleepwalking and/or sleep terrors. ${ }^{20,40,42,44,45}$ On the other hand, three studies included participants exclusively with sleepwalking ${ }^{34,35,43}$ and one with sleep terrors. ${ }^{41}$

Almost all the studies recruited subjects who did not use medications. ${ }^{20,34,35,41-45}$ Only one study did not report this information. ${ }^{40}$

Four studies included a healthy control group matched for sex and age with the parasomniac group. ${ }^{20,34,35,41}$ However, Pilon et $\mathrm{al}^{35}$ and Guilleminault et $\mathrm{al}^{34}$ did not consider the control group when evaluating EEG patterns before the BEp.

All participants underwent overnight PSG assessments. $^{20,34,35,40-45}$ As detailed in Table 2, seven studies used an undisturbed night protocol. Conversely, two works ${ }^{35,42}$ applied a sleep-deprivation protocol that included a baseline night and a recovery night.

Table 2 shows the number and the typology of BEp recorded. All patients showed at least one BEp during EEG recordings. ${ }^{20,34,40-45}$ Subjects that did not exhibit BEp were excluded from the analyses. ${ }^{35}$ All studies analyzed the BEp that occurred during SWS.

The authors recorded only BEp of sleepwalking and sleep terrors, characterized by different types of behaviors with a distinct level of complexity. The most straightforward 
Table 2 Sample Characteristics and Night Recordings Description

\begin{tabular}{|c|c|c|c|c|c|c|c|c|c|c|}
\hline \multirow{4}{*}{ Ref } & \multicolumn{5}{|c|}{ Sample Characteristics } & \multicolumn{5}{|c|}{ Night Recordings } \\
\hline & \multicolumn{4}{|c|}{ Parasomnia Group } & \multirow{3}{*}{$\begin{array}{l}\text { Control } \\
\text { Group }\end{array}$} & \multirow{3}{*}{$\begin{array}{l}\text { Experimental } \\
\text { Night }\end{array}$} & \multicolumn{4}{|c|}{ Activations Characteristics } \\
\hline & \multirow[t]{2}{*}{$\mathbf{N}$} & \multirow[t]{2}{*}{ Gender } & \multirow[t]{2}{*}{$\begin{array}{l}\text { Mean Age } \\
\text { (Years) (sd) }\end{array}$} & \multirow[t]{2}{*}{$\begin{array}{l}\text { Parasomnia } \\
\text { Diagnosis }\end{array}$} & & & \multicolumn{2}{|c|}{$\begin{array}{l}\text { Behavioral } \\
\text { Episodes of } \\
\text { NREM } \\
\text { Parasomnias } \\
\text { (BEp) }\end{array}$} & \multicolumn{2}{|c|}{$\begin{array}{c}\text { Non-Behavioral } \\
\text { Arousals } \\
\text { (NBEp) }\end{array}$} \\
\hline & & & & & & & $\mathbf{N}$ & $\begin{array}{c}\text { Episode } \\
\text { Onset }\end{array}$ & $\mathbf{N}$ & $\begin{array}{c}\text { Episode } \\
\text { Onset }\end{array}$ \\
\hline $\begin{array}{l}\text { Schenck } \\
\text { et } \mathrm{al}^{40}\end{array}$ & $38 *$ & $F=17$ & $28.9(9.6)$ & $\begin{array}{l}\mathrm{SW}=3 \\
\mathrm{SW} \text { and } \\
\mathrm{ST}=35\end{array}$ & - & $\begin{array}{l}\text { I or } 2^{* *} \\
\text { Undisturbed night }\end{array}$ & 87 & sWs & 163 & sWs \\
\hline Zadra et $\mathrm{al}^{41}$ & 1 & $\mathrm{~F}$ & 32 & $\mathrm{ST}=\mathrm{I}$ & $\begin{array}{c}\mathrm{N}^{\circ} 10(\mathrm{~F}=5) \\
\text { Age: } \\
\text { matched }\end{array}$ & $\begin{array}{l}\text { P: } 3 \text { undisturbed } \\
\text { nights } \\
\text { C: } 2 \text { undisturbed } \\
\text { nights }\end{array}$ & 3 & sWs & - & - \\
\hline Espa et $\mathrm{al}^{20}$ & 11 & $F=6$ & $31.2(2.24)$ & $\begin{array}{c}\mathrm{SW}=3 \\
\mathrm{ST}=6 \\
\mathrm{SW} \text { and } \mathrm{ST}=2\end{array}$ & $\begin{array}{l}\mathrm{N}^{\circ} \mathrm{II} \\
\text { (matched) } \\
\text { Mean age: } \\
32.1(2.48)\end{array}$ & $\begin{array}{l}\text { P: I undisturbed } \\
\text { night } \\
\text { C: I undisturbed } \\
\text { night }\end{array}$ & 15 & sWs & $\begin{array}{l}P=15 \\
C=40\end{array}$ & $\begin{array}{l}P=S W S \\
C=S W S\end{array}$ \\
\hline $\begin{array}{l}\text { Guilleminault } \\
\text { et } \mathrm{al}^{34}\end{array}$ & 12 & $F=7$ & $23.8(3)$ & $S W=12$ & - & $\begin{array}{l}\text { I Undisturbed } \\
\text { night }\end{array}$ & 21 & sWs & - & - \\
\hline Pilon et $\mathrm{al}^{35}$ & 10 & $F=7$ & $25.1(4.1)$ & $S W=10$ & - & $\begin{array}{l}\text { Sleep-deprivation } \\
\text { protocol (h38): } \\
\text { I Baseline night } \\
\text { I Recovery }\end{array}$ & 35 & sWs & - & - \\
\hline Jaar et $\mathrm{al}^{42}$ & 22 & $F=11$ & $28.6(7.4)$ & $\begin{array}{l}\text { SW and/or } \\
\text { ST }=22\end{array}$ & - & $\begin{array}{c}\text { Sleep-deprivation } \\
\text { protocol (h25): } \\
\text { I Baseline } \\
\text { I Recovery }\end{array}$ & 22 & SWS & - & - \\
\hline $\begin{array}{l}\text { Perrault } \\
\text { et } \mathrm{al}^{43}\end{array}$ & 12 & $F=9$ & $27.4(8.4)$ & $S W=12$ & - & $\begin{array}{l}\text { I Undisturbed } \\
\text { night }\end{array}$ & 12 & SWS & 12 & sWS \\
\hline $\begin{array}{l}\text { Januszko } \\
\text { et } \mathrm{al}^{44}\end{array}$ & 20 & $\mathrm{~F}=4$ & $\begin{array}{l}\text { Age range: } \\
\text { 18-38 }\end{array}$ & $\begin{array}{l}S W=7 \\
S W \text { and } \\
S T=13\end{array}$ & - & $\begin{array}{l}\text { I Undisturbed } \\
\text { night }\end{array}$ & 26 & SWS & - & - \\
\hline $\begin{array}{l}\text { Desjardins } \\
\text { et } \mathrm{al}^{45}\end{array}$ & 27 & $F=14$ & $29(7.6)$ & $\begin{array}{l}\text { SW and/or } \\
\text { ST }=27\end{array}$ & - & $\begin{array}{l}\text { I Undisturbed } \\
\text { night }\end{array}$ & 27 & SWS & - & - \\
\hline
\end{tabular}

Notes: The "Sample Characteristics" column reports the description of parasomniac and control group. The "Night Recordings" column details the numbers of experimental night and the description of night protocol. The "Activation Characteristics" column describes the behavioral episodes of NREM parasomnia (BEP) and Nonbehavioral arousals (NBEp) features, specifically the number of BEp and the BEp's onset by sleep stage. *Schenck et al ${ }^{40}$ recruited the 38 subjects' PSG from a storage room. **S Schenck et a $\mathrm{l}^{40}$ reported that some participants performed 2 consecutive nights while others only I night.

Abbreviations: F, female; sd, standard deviation; SW, sleepwalking; ST, sleep terrors; P, parasomniac group; C, control group; SWS, slow wave sleep.

behaviors observed were as follows: sitting in the bed, moving arms, kneeling, and verbal utterances. ${ }^{20,34,40,41,43,44}$ The most elaborate behaviors recorded were leaving the bed ${ }^{44}$ or trying to get out of bed. ${ }^{43}$ Guilleminault et $\mathrm{al}^{34}$ also reported that all the somnambulistic episodes were associated with a confusional state. One study ${ }^{35}$ evaluated the complexity of BEp using a 3-point scale: ${ }^{12}$ type 1 (eg, turning and resting on one's hands, playing with the bedsheets), type 2 (sitting 
up, kneeling, or trying to get out of bed), and type 3 (leaving the bed). In this case, the authors observed that sleep deprivation increased the BEp's frequency and complexity. Indeed, the most elaborate BEp (types 2 and 3) occurred exclusively during the recovery night.

\section{Investigation of Electroencephalographic} Patterns Before Behavioral Episodes

The analysis of sleep EEG patterns preceding the onset of the BEp is the key feature of these studies. The authors used different approaches: EEG visual scoring, quantitative EEG analysis (all EEG power spectra were computed using the Fast Fourier Transform [FFT]), EEG functional connectivity, and source localization. All studies selected specific time windows chosen according to the objective of the investigation (see Table 3).

Most studies aimed to investigate the possible role of sleep EEG patterns during SWS during the onset of BEp. Table 4 summarizes the main findings.

\section{Visual Scoring}

As shown in Table 3, two studies examined the sleep EEG variables through visual scoring. Schenck et al ${ }^{40}$ focused on "delta-wave clusters" and "delta-wave build-up." Besides these two activities, Pilon and colleagues ${ }^{35}$ also evaluated the HSD.

Schenck et $\mathrm{al}^{40}$ compared two consecutive epochs of BEp and non-behavioral arousals (NBEp). The scoring criteria of the NBEp are the following: "any increase in electromyography (EMG) on any channel, which is accompanied by a change in pattern on any additional channel." ${ }^{, 46}$ The authors observed no differences between 0-10 seconds and 11-30 seconds in delta-wave build-up and cluster, assessed at C3 before BEp and NBEp. Moreover, the authors found a low presence of deltawave build-up and cluster before BEp and NBEp.

Pilon et $\mathrm{al}^{35}$ found similar results during baseline and recovery nights for any considered channels (F3, C3, P3, T3, O1).

The main findings were observed in the time spent in HSD during the 5 minutes preceding the BEp. ${ }^{35}$ Indeed, the HSD's presence in the frontal and central derivations was significantly higher during recovery than in the baseline night, but it was distributed equally during the 5-minute timeline. ${ }^{35}$

On the other hand, the proportions of HSD occurring during the last 10 seconds $(0-10$ seconds) and the last 30 seconds $(0-30$ seconds) were not different between baseline and recovery nights on any leads.

Lastly, Pilon et $\mathrm{al}^{35}$ examined the proportion of BEp preceded by the considered sleep parameters (see Table 3) as a function of complexity of BEp for each channel, but no significant difference was observed during simple and complex BEp. ${ }^{35}$

\section{Quantitative EEG Analyses}

Four studies focused on quantitative measures of sleep intensity and depth. Hence, the FFT analyses were performed to measure the SWA and delta activity considering the following ranges of frequency: $0.50 \mathrm{~Hz}-4.50 \mathrm{~Hz}^{42,43}$ or $0.75-4.50,{ }^{20}$ and $1.00-4.00 \mathrm{~Hz} .{ }^{42,43}$ Specifically, as shown in Table 3, the authors analyzed different sub-bands: a) slow delta $(0.50-1.00 \mathrm{~Hz})^{42,43}$ and b) low $(0.75-2.00 \mathrm{~Hz})$ and high $(2.25-4.00 \mathrm{~Hz})$ delta activity. ${ }^{34}$

Lastly, two studies ${ }^{42,43}$ performed the automatic detection of slow oscillations ([1] negative peak $<-40 \mu \mathrm{V}$, [2] peak-to-peak amplitude $>75 \mu \mathrm{V}$, [3] duration of negative deflection $>125 \mathrm{~ms}$ and $<1500 \mathrm{~ms}$, and [4] duration of positive deflection $<1000 \mathrm{~ms}),{ }^{42,43}$ or very slow oscillations ([1] negative peak $<-80 \mu \mathrm{V}$, [2] peak-to-peak amplitude $>140 \mu \mathrm{V}$, [3] duration of negative deflection $>125$ $\mathrm{ms}$ and $<1500 \mathrm{~ms}$, and [4] duration of positive deflection $<1000 \mathrm{~ms}) .{ }^{42}$ However, the detected activity, using the first criteria, mostly overlaps with SWA. Thus, we consider as slow oscillation (SO), in accordance with the current literature $^{47-50}$ (ie, SO: EEG $>140 \mu \mathrm{V},<1 \mathrm{~Hz}$ ), the activity defined by Jaar et $\mathrm{al}^{42}$ as "very slow oscillation."

Firstly, Espa et $\mathrm{al}^{20}$ analyzed three- time windows (see Table 3), comparing BEp and NBEp, and also performed a time course dividing 10 minutes into ten epochs of 60 seconds, only before BEp on C3 and C4. Moreover, the authors included a control group to evaluate differences with the parasomniac group's NBEp. ${ }^{20}$ The NBEp were defined as a) an abrupt shift in EEG frequency which may include theta, alpha and/or frequencies greater than $16 \mathrm{~Hz}$ (but no spindles), b) at least 10 seconds of continuous sleep were required prior to the NBEp, c) the arousal reaction must last for at least 3 seconds and for less than 15 seconds, d) the occurrence of simultaneous EMG activity together with the arousal reaction was necessary in REM sleep only."20,51

The main finding was a gradual increase of SWA over the last 10 minutes before the BEp. ${ }^{20}$ Specifically, they recorded the highest level of SWA in the last 2 minutes. The mean SWA value was also significantly higher during 


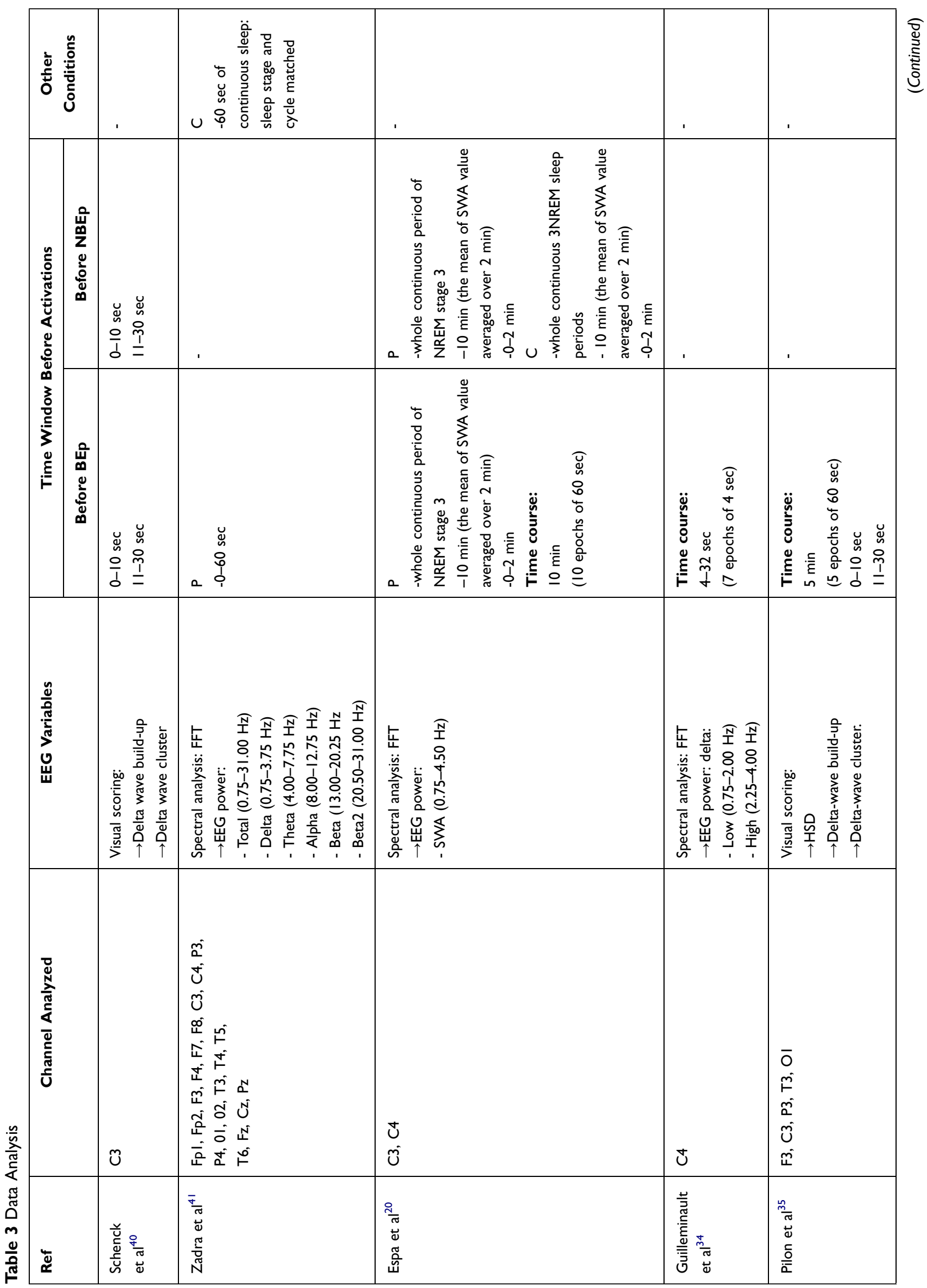




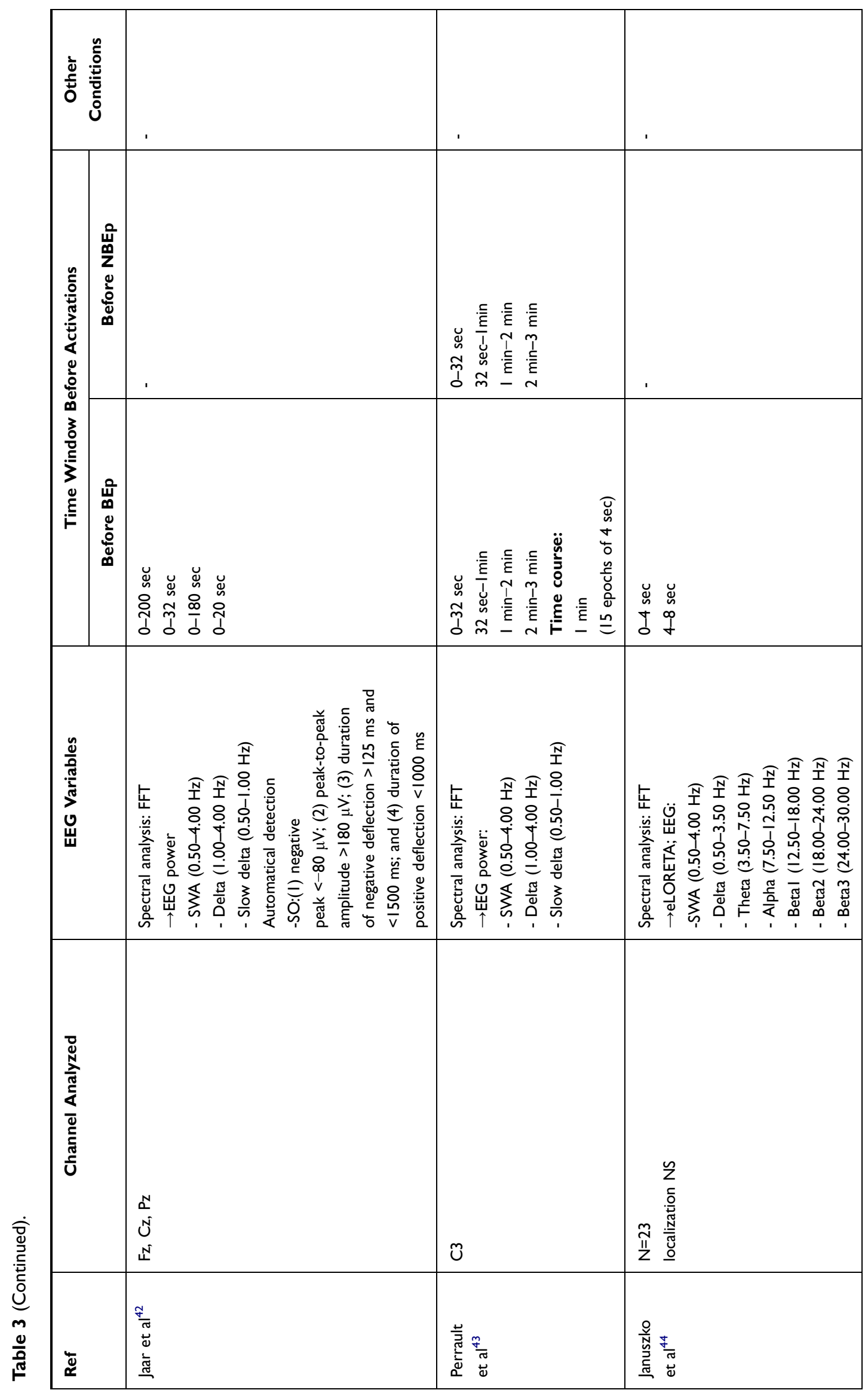




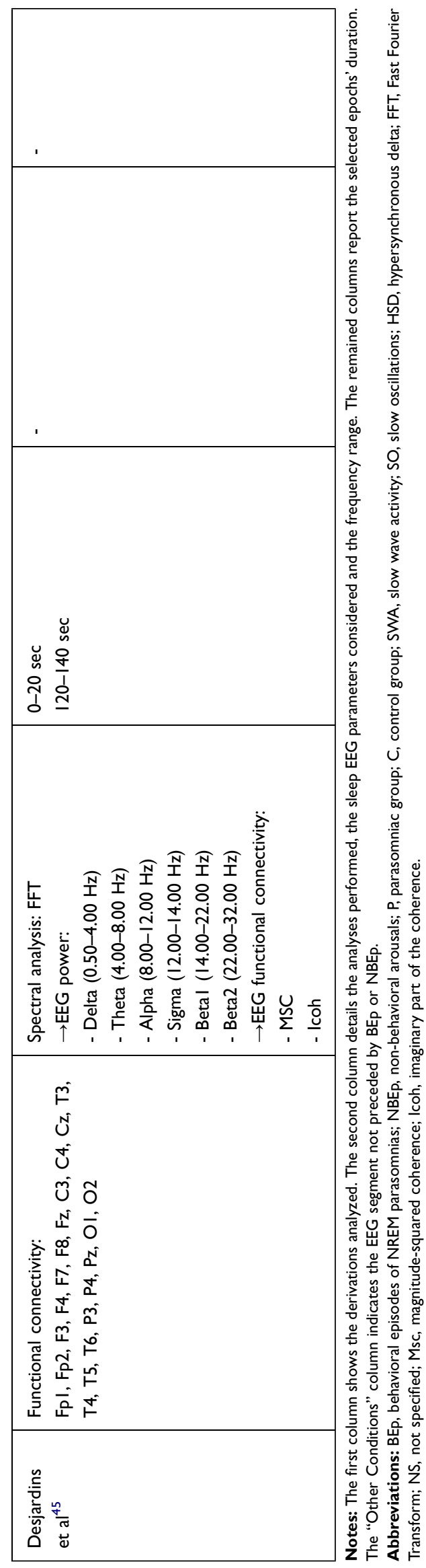

the 2 minutes immediately before the onset of the BEp, compared with the two longer time windows. Moreover, a significant difference in the mean SWA value was found between EEG segments before BEp and NBEp. No differences were found between parasomniac and control groups in the three-time windows preceding the NBEp.

Jaar et $\mathrm{al}^{42}$ found similar results when assessing the variation in the EEG activity over the 200 seconds (0-200 seconds) and the last 32 seconds ( $0-32$ seconds) before the BEp on the midline. The authors also compared the last 20 seconds before the BEp with the previous 180 seconds.

Overall, the main findings showed a significant linear increase of SWA, delta, and slow delta during the 32 seconds immediately before the BEp, particularly on $\mathrm{Cz}$ and Pz. The same analyses conducted over the $200 \mathrm{sec}-$ onds did not reveal any significant trend for SWA, delta, and slow delta. Moreover, the authors found a higher EEG power for SWA and delta in the last 20 seconds on frontal lead than in the previous 180 seconds. ${ }^{42}$ Consistently, SO density showed similar results on the frontal and central location. Instead, SO amplitude increased during the 32 seconds immediately before the BEp only on $\mathrm{Fz}^{42}$ Different to Espa et $\mathrm{al}^{20}{ }^{20} \mathrm{Jaar}$ et $\mathrm{al}^{42}$ found that such an increase was restricted to the last seconds before the BEp.

Lastly, Guilleminault et $\mathrm{al}^{34}$ investigated the low and high delta time course over the 32 seconds before the BEp on $\mathrm{C} 4$. Consistent with Jaar et $\mathrm{al}^{42}$ the authors revealed an increase of low delta during the last seconds (from 12 to 8 seconds and from 8 to 4 seconds) closer to BEp than an earlier period (from 32 to 28 seconds). ${ }^{34}$

In line with other studies, ${ }^{20,40}$ Perrault et $\mathrm{al}^{43}$ included the NBEp in the analyses, defined as follows: "a transient interruption of sleep, identifiable when $\geq 50 \%$ of an epoch contained alpha $(8-13 \mathrm{~Hz})$ activity or low-voltage, mixed $(2-7 \mathrm{~Hz})$ frequency activity." 52 In this case, time courses were analyzed over 3 minutes before BEp and NBEp (3-2 minutes before episodes, 2- 1 minute, 1 minute to 32 seconds, and 32 seconds to episode onset) and over the last minute (15 windows of 4 seconds) on the central channel (C3). However, the authors reported controversial findings. ${ }^{43}$ Indeed, they did not observe a progressive increase of EEG activity over the 3 minutes and the last minute before the BEp, but the sleep parameters considered (ie, SWA, delta and slow delta) were always higher before the somnambulistic episodes than NBEp. ${ }^{43}$.

Finally, three studies performed EEG power analyses on sleep intervals before the BEp, for different frequency bands (ie, delta, theta, alpha, sigma, and beta bands) across 
Table 4 Main EEG Findings: The Main Results are Divided for EEG Sleep Parameters Investigated

\begin{tabular}{|c|c|c|c|}
\hline & EEG Investigation & Ref & EEG Finding \\
\hline \multirow[t]{3}{*}{$\begin{array}{l}\text { Visual } \\
\text { scoring }\end{array}$} & \multirow[t]{2}{*}{$\begin{array}{l}\text { Delta-wave build-up* } \\
\text { Delta-wave cluster** }\end{array}$} & $\begin{array}{l}\text { Schenck } \\
\text { et } \mathrm{al}^{40}\end{array}$ & No difference between $0-10 \mathrm{sec}$ and $1 \mathrm{I}-30 \mathrm{sec}$ before BEp and NBEp \\
\hline & & Pilon et $\mathrm{al}^{35}$ & $\begin{array}{l}\text { No difference between } 0-10 \mathrm{sec} \text { and } \mathrm{II}-30 \mathrm{sec} \text { before the BEp during baseline and recovery nights and as } \\
\text { a function of complexity episodes }\end{array}$ \\
\hline & $\begin{array}{l}\text { Hypersynchronous delta (HSD) } \\
\text { *** }\end{array}$ & Pilon et $\mathrm{al}^{35}$ & $\begin{array}{l}\text { - Higher HSD prevalence during } 5 \text { min before BEp during recovery than baseline night on central leads } \\
\text { - No difference in the proportion of BEp preceded by HSD-10 and HSD-30 sec between baseline and } \\
\text { recovery nights and as a function of episodes' complexity on any leads }\end{array}$ \\
\hline \multirow[t]{9}{*}{$\begin{array}{l}\text { Quantitative } \\
\text { EEG }\end{array}$} & \multirow[t]{4}{*}{$\begin{array}{l}\text { Slow-wave activity (SWA), } \\
\text { delta waves and sub-bands }\end{array}$} & Espa et $\mathrm{al}^{20}$ & $\begin{array}{l}\text { - Higher SWA during 0-2min before BEp than during } 10 \mathrm{~min} \text { and whole NREM sleep stage } 3 \text { before BEp } \\
\text {-Higher SWA during all time windows before the BEp than the three-time windows before NBEp, in } P \\
\text {-No difference between } P \text { and } C \text { in the mean SWA value before NBEp } \\
\text { Time course: significant gradual increase of SWA over the } 10 \text { min before BEp }\end{array}$ \\
\hline & & $\begin{array}{l}\text { Guilleminault } \\
\text { et } \mathrm{al}^{34}\end{array}$ & $\begin{array}{l}\text { - Higher low delta during 4-8 and 8-12 sec than } 28-32 \text { sec before BEp } \\
\text {-No difference in higher delta range over the } 32 \mathrm{sec} \text { before BEp }\end{array}$ \\
\hline & & Jaar et $\mathrm{al}^{42}$ & $\begin{array}{l}\text { - Significant increase in SWA, delta and slow delta over the } 32 \mathrm{sec} \text { before BEp on the midline } \\
\text { - Higher SWA and delta over the } 20 \mathrm{sec} \text { before BEp than previous } 180 \mathrm{sec} \text { on Fz and Cz } \\
\text { - No difference in slow delta between } 20 \mathrm{sec} \text { before BEp and previous } 180 \mathrm{sec} \\
\text { - No significant trend of SWA delta and slow delta over the } 200 \mathrm{sec} \text { before BEp }\end{array}$ \\
\hline & & $\begin{array}{l}\text { Perrault } \\
\text { et } \mathrm{al}^{43}\end{array}$ & $\begin{array}{l}\text { - Higher SWA, delta and slow delta before BEp than NBEp } \\
\text { - No difference of SWA, delta and slow delta between the } 3 \text { time-windows (0-32 sec; } 32 \mathrm{sec}-1 \text { min; I-2 } \\
\text { min; 2-3 min) before BEp and NBEp. } \\
\text { Time course: } \\
\text { - Higher SWA, delta and slow delta during I min before BEp than NBEp. } \\
\text { - No significant increase of SWA, delta and slow delta over the I min before BEp on NBEp. }\end{array}$ \\
\hline & Slow Oscillation (SO) & Jaar et $\mathrm{al}^{42}$ & $\begin{array}{l}\text { SO density: } \\
\text { - Significant linear increase over the } 200 \mathrm{sec} \text { before BEp on FZ and } \mathrm{Cz} \\
\text { - Significant linear increase over the } 32 \mathrm{sec} \text { before BEp on Fz } \\
\text { - Higher over the } 20 \mathrm{sec} \text { before BEp than previous } 180 \mathrm{sec} \text {, respectively on } \mathrm{Fz} \text { and } \mathrm{Cz} \\
\text { SO amplitude: } \\
\text {-No significant trend over the } 200 \mathrm{sec} \text { before BEp } \\
\text { - Significant linear increase of SO amplitude over the } 32 \mathrm{sec} \text { before BEp on FZ } \\
\text { - No difference between } 20 \mathrm{sec} \text { before BEp and previous } 180 \mathrm{sec}\end{array}$ \\
\hline & \multirow[t]{2}{*}{ Canonical EEG bands } & Zadra et $\mathrm{al}^{4 I}$ & $\begin{array}{l}\text {-Higher delta during } 60 \mathrm{sec} \text { before } \mathrm{BEp} \text { in } \mathrm{P} \text { than } \mathrm{C} \text {, in central and frontal areas } \\
\text { - Higher delta in left regions than right frontal areas } \\
\text {-Higher delta during most intense ST than less intense }\end{array}$ \\
\hline & & $\begin{array}{l}\text { Desjardins } \\
\text { et } \mathrm{al}^{45}\end{array}$ & $\begin{array}{l}\text {-Higher delta during } 20 \mathrm{sec} \text { before BEp than } 20 \mathrm{sec} \text { occurring } 2 \text { min before BEp on Fz } \\
\text {-Higher theta during } 20 \mathrm{sec} \text { before BEp than } 20 \mathrm{sec} \text { occurring } 2 \mathrm{~min} \text { before BEp on } \mathrm{Fz}\end{array}$ \\
\hline & Source Localization (eLoreta) & $\begin{array}{l}\text { Januszko } \\
\text { et } \text { al }^{44}\end{array}$ & $\begin{array}{l}\text {-Higher beta }(24.0-30.0 \mathrm{~Hz}) \text { during } 0-4 \mathrm{sec} \text { than } 4-8 \mathrm{sec} \text { before BEp in } 2 \text { voxels (Brodmann area } 33 \text { and } \\
\text { 24) }\end{array}$ \\
\hline & Functional Connectivity & $\begin{array}{l}\text { Desjardins } \\
\text { et } \mathrm{al}^{45}\end{array}$ & $\begin{array}{l}\text { Msc } \\
\text { - No difference between } 20 \mathrm{sec} \text { before BEp than } 20 \mathrm{sec} \text { occurring } 2 \text { min before BEp } \\
\text { ICoh } \\
\text { - Lower low delta functional connectivity during } 20 \mathrm{sec} \text { before BEp than } 20 \mathrm{sec} \text { occurring } 2 \text { min before } \\
\text { BEp for parietal and occipital regions } \\
\text {-Higher alpha functional during } 20 \mathrm{sec} \text { before BEp than } 20 \mathrm{sec} \text { occurring } 2 \text { min before BEp connectivity for } \\
\text { frontal and parietal regions } \\
\text {-Higher beta functional connectivity during } 20 \mathrm{sec} \text { before BEp than } 20 \mathrm{sec} \text { occurring } 2 \text { min before BEp for } \\
\text { fronto-temporal, parietal and occipital regions. }\end{array}$ \\
\hline
\end{tabular}

Notes: The visual scoring criteria of the EEG activities in the first row are in according to Schenck et al ${ }^{40}$ and Pilon et al ${ }^{35}$. $*$ The delta-wave build-up are defined as the highest-amplitude delta wave had to occur in the 10 seconds immediately preceding a BEp, as compared with the previous II- to 30-second. **The delta-wave cluster is defined as two or more consecutive highest-amplitude delta waves occurring in the 10 seconds immediately preceding a BEp. ***The Hypersynchronous delta (HSD) is defined as the presence of at least 5 seconds of continuous high-voltage $(\geq I 50 \mu \mathrm{V})$ delta waves $(\mathrm{I}-3 \mathrm{~Hz})$ immediately preceding a BEp. The minimal interval needed without HSD to score two consecutive HSD events was at least I second.

Abbreviations: HSD, hypersynchronous delta; BEp, behavioral episodes of NREM parasomnias; NBEp, non-behavioral arousals; P, parasomniac group; C, control group; magnitude-squared coherence; Icoh, imaginary part of the coherence. 
the whole scalp. Specifically, the authors focused on the distribution of EEG power bands ${ }^{41,45}$ or the neural networks involved with the onset of the BEp. ${ }^{44,45}$

Zadra et $\mathrm{al}^{41}$ considered 60 seconds before the BEp compared to an EEG segment of healthy subjects, chosen from the same sleep cycle and stage. They analyzed the topographic distribution on 19 scalp derivations (see Table 3), revealing higher delta power on frontal and central areas during 60 seconds before the onset of sleep terrors compared to the control group. This difference was greater in the left than right regions. Finally, the most intense sleep terror episode was characterized by higher delta power than the least intense.

Januszko et $\mathrm{al}^{43}$ investigated the source localization of the BEp using 23 EEG channels. The authors observed higher beta frequency $(24.0-30.0 \mathrm{~Hz})$ during the last 4 seconds than in the 4-8 seconds before the onset of the nocturnal BEp in anterior cingulate cortices (Brodmann areas 33 and 24).

Lastly, Desjardins et $\mathrm{al}^{45}$ performed a power analysis of six frequency EEG bands (see Table 3) and the functional connectivity analysis using the magnitude-squared coherence (Msc) and the imaginary part of the coherence (ICoh) on 19 derivations. In this case, the authors compared two equal EEG segments: one immediately before the onset of BEp and the second one occurring 2 minutes prior to the BEp. The authors revealed a significant increase in delta and theta activity during the 20 seconds preceding the episodes, compared to a similar segment occurring 2 minutes before the BEp, exclusively on Fz. Moreover, the functional connectivity analysis showed no significant differences between the two conditions. ${ }^{45}$ However, the analyses with ICoh presented significant differences between the two conditions for low delta $(0.10-2.00 \mathrm{~Hz})$, alpha (9.00-11.00 $\mathrm{Hz}$ ), and beta $(22.00-24.00 \mathrm{~Hz} ; 26.00-29.00 \mathrm{~Hz})$ EEG activity. Parietal and occipital regions showed lower functional connectivity in the low delta band during the experimental condition compared to the baseline. On the contrary, greater functional connectivity was observed between the frontal and parietal regions in the alpha band. Similarly, the beta band showed higher connectivity for symmetric interhemispheric networks, including fronto-temporal, parietal, and occipital regions. ${ }^{45}$

\section{Discussion}

This systematic review analyzed sleep EEG patterns immediately before the BEp to shed light on the mechanism underlying the onset of nocturnal behaviors.
The findings related to deep sleep showed an increase in SWA/delta, slow delta, and SO in the epochs closer to the BEp in frontal ${ }^{42,45}$ and central $^{20,34,42}$ areas. Specifically, the enhancement of slow frequency activities seems restricted to the last 30 seconds before the BEp, ${ }^{42,45}$ reaching the highest peak in the last 10 seconds (ie, low delta $[0.75-2.00 \mathrm{~Hz}]$ was highest in the 4-12 sec time window). ${ }^{34}$ Moreover, the analysis of the topographical distribution of canonical EEG power provided results consistent with those mentioned above. ${ }^{41,45}$

One interpretation of these results suggests that many arousals during the night may enhance the sleep pressure, increasing slow-frequency activity. ${ }^{20,34,42,45}$ A feature of somnambulism is the abnormal response to internal or external stimulation. Indeed, several factors (eg, stress, external noises, anxiety, obstructive sleep apneas, periodic leg movements, etc), 9,27,53 which typically fragment sleep, are associated with the onset of somnambulistic episodes in predisposed individuals. Thus, the abnormal and frequent somnambulistic responses and the high frequency of spontaneous arousals in the parasomnia group may cause greater sleep pressure. In other words, the increase of slow frequency activity may be considered an expression of the homeostatic process. Therefore, the SWA and SO enhancement may represent the brain's attempt to maintain the deep sleep against the abnormal sleep interruption, ${ }^{54}$ limiting the thalamocortical activation through the corticothalamic inhibition feedback. ${ }^{34,42,43,55,56}$

These results are consistent with HSD's topographical findings. ${ }^{35,40}$ In this respect, Pilon et $\mathrm{al}^{35}$ reported a frontocentral gradient of HSD, in line with early findings. ${ }^{10,33,40,57}$ However, low specificity of HSD before the BEp was observed, ${ }^{35,40}$ supporting the idea that it was not a marker of BEp. ${ }^{35,40}$ Indeed, as mentioned before, HSD was also observed in other conditions that presented sleep fragmentation. $^{32}$ However, studies concerning HSD are scarce, and the role of HSD remains unclear to date. ${ }^{35}$

On the other hand, the BEp is associated with factors that promote deep sleep, such as sleep deprivation. ${ }^{58,59}$ Moreover, the whole-night studies showed an anomalous expression of SWS in participants with somnambulism. ${ }^{11,21,22}$ Thus, predisposing factors might promote SWS dysfunction, which might play a role in the onset of BEp. ${ }^{13,31,60}$ In this view, the combination of predisposing factors that dysregulate deep sleep and factors that induce sleep fragmentation could trigger BEp in predisposed individuals. $^{42,43,58,61}$ 
Only one study observed an unexpected result, reporting no increase of SWA immediately before the BEp. ${ }^{43}$ Examining only one central channel might have biased these findings, overlooking information about the topographical distribution of SWA. Indeed, other studies have observed that the increase of SWA was prevalent in frontal derivation. ${ }^{41,42,45}$

EEG functional connectivity or source localization adds information about neural networks involved in the onset of BEp. The source localization analysis revealed an increase in the beta frequency band during the last 4 seconds in the anterior cingulate cortices before the BEp. ${ }^{44}$ Thus, Januszko et $\mathrm{al}^{44}$ suggested that arousalrelated disinhibition of cortical areas involved in motor control could explain the motor episodes. It is thought that the cingulate cortex would play a crucial role in the planning and initiation of motor activity. ${ }^{62-64}$ However, it is unknown whether these regions are implicated in intentional movements. ${ }^{63}$ The results through functional EEG connectivity supplement information about the dynamic of the BEp onset. In this vein, Desjardins et $\mathrm{al}^{45}$ found a local connectivity decrease in the parietal and occipital delta frequency band. Instead, the anteroposterior bilateral networks showed a long-range connectivity increase in the high-frequency bands (ie, alpha and beta). The authors suggested that such functional connectivity changes express the transition toward wakefulness or lighter sleep. ${ }^{45}$ Hence, the increase of delta power observed by early studies could represent a more complex mechanism rather than a brain's attempt to maintain deep sleep. ${ }^{45}$ Therefore, a gradual and complex arousal process, occurring in particular over posterior areas, could precede the BEp. $^{45}$

These results are consistent with stereo-EEG and wakefulness studies. ${ }^{7,36,44,45,65,66}$ Indeed, fast activity (ie, $25 \mathrm{~Hz}$ ) in the motor and limbic structures (central cingulate cortex, insular cortex, temporopolar cortices, and amygdala) and an increased delta band in the frontal and parietal dorsolateral associative cortices were observed before confusional arousals. ${ }^{65,66} \mathrm{~A}$ recent study that included a sample of participants with confusional arousals found some heterogeneous results. Although the coexistence of sleep-wake patterns was observed, the authors did not find high-frequency activity in most of the cingulate cortex. ${ }^{67}$

Overall, these findings showed the coexistence of SWA in the frontal regions and wake-like EEG patterns in the motor areas immediately before BEp. ${ }^{44,45,65}$ Consistent with the local sleep concept, ${ }^{68}$ DOAs simultaneously exhibit sleep and wake patterns in specific brain areas. ${ }^{68}$ In this perspective, DOAs could be characterized by a "dissociation state", between the cingulate cortex, which could determine the BEp, and the central and frontal areas. ${ }^{7,13,65,66}$ Thus, Castelnovo et $\mathrm{al}^{7}$ defined NREM sleep parasomnias as sleep-state dissociation disorders. To date, the sleep-state dissociation hypothesis seems promising because it might clarify the mechanism underlying behavioral and cognitive dissociation during BEp.

\section{Methodological Issue: Insights for Future Directions}

The second aim of the systematic review was to provide new insight for future research by examining the principal methods used to investigate the EEG patterns of BEp.

The main issue of works based on visual scoring is the absence of reliable criteria for identifying and quantifying HSD. ${ }^{35,39,56}$ The use of EEG power analysis and the whole-scalp examination better define the nature of HSD and, more directly, can clarify the modulation of delta activity in BEp.

Other issues concern the definition of the band frequency range. The studies reviewed did not use homogeneous criteria for SWA, ${ }^{20,34,41-43}$ delta activity, ${ }^{20,34,41-43}$ and SO. ${ }^{42,43}$ Therefore, the use of different criteria in the definition of EEG frequency range could explain the partially inconsistent findings.

Furthermore, some characteristics of the sample recruitment might influence the obtained results. The copresence of different parasomnias (ie, sleepwalking and sleep terrors $)^{20,40,42,44,45}$ and the phenotypic variations within the same condition (eg, age of onset, episode frequency, episode complexity, etc. $)^{14,32,43}$ might explain the different SWA trend observed before the BEp. ${ }^{20,42,43}$ Although these conditions may share the same pathophysiological features, future studies should examine more homogeneous samples as a function of these variables.

Moreover, all samples investigated included adults or young adults. It is known that NREM parasomnias are age-related. ${ }^{69-71}$ Besides, sleep changes during the life span, particularly concerning SWA, are associated with brain maturation. ${ }^{72,73}$ Thus, transversal and longitudinal studies should be performed to identify eventual agerelated differences in EEG patterns before the BEp.

Another methodological issue to consider is the presence/absence of a control group. The choice of a control 
group or control condition is challenging in investigations of BEp. Although the control group ensures an adequate comparison of the whole-night macrostructural parameters, it is impossible to analyze the EEG patterns before the BEp in healthy subjects, because they do not experience such activations. Zadra et $\mathrm{al}^{41}$ selected the EEG segment from the same cycle and sleep stage in the control group to solve this issue. However, a single time window does not provide sufficient information about the dynamic of EEG power changes before the BEp. Moreover, it is worth noting that these analyses were performed on only one subject. ${ }^{41}$

Differently, three studies evaluated the NBEp, ${ }^{20,40,43}$ which were considered as a control condition.

Moreover, the literature reports a large prevalence of microarousals recorded before the BEp. ${ }^{30,34}$ Therefore, it would be interesting to investigate if the arousals could trigger the abnormal activations. ${ }^{34}$

Recent studies proposed that lower spindle density during SWS in sleepwalkers than in the control group reflects a vulnerability to external stimuli, ${ }^{20}$ which increases the occurrence of BEp. ${ }^{74}$ Hence, sleep spindles can also be involved in the onset of BEp. Studies directly aimed to assess the functional role of sleep spindles in the onset of BEp are needed. Moreover, there were observed abnormalities in K-complex morphology in pediatric parasomniac groups. ${ }^{75}$ The K-complex might have a sleep protective role. ${ }^{76-80}$ However, few studies have investigated the role of K-complex in NREM parasomnia, so future research could evaluate the K-complex characteristics before BEp.

As mentioned before, it is recognized that sleep deprivation improves the frequency and complexity of parasomnia activations. $^{12,35,53,58,81}$ Therefore, the SWA and SO enhancement, observed before the BEp by Jaar et $\mathrm{a}^{42}$ could not be generalizable due to the typical increase in slow wave frequencies during recovery nights after sleep deprivation. ${ }^{82}$ In contrast, Pilon et $\mathrm{al}^{35}$ compared the baseline night with the recovery nights, but no EEG power analysis was carried out.

To date, it is not clear if SWS dysfunction induces BEp or whether sleep pressure and fragmentation provoke the increase of slow frequency activity. Thus, protocols of sleep deprivation and/or auditory stimulation protocol might shed light on the processes involved in the BEp.

One of the main features of the reviewed studies is the comparison of EEG segments of different time windows, ${ }^{20,34,35,40-45}$ obtaining results regarding the temporal distribution of EEG activities. However, it is necessary to broaden research in this field to clarify the inconsistent findings mentioned above. The investigation of closer time windows (ie, from 0 to 30 seconds) before the BEp, through source localization or functional connectivity, would seem promising. In this way, it is possible to obtain information about the networks involved in the onset of BEp with a high spatial and temporal resolution. The first studies that used these approaches revealed greater EEG activity in high-frequency bands in networks involved in motor planning. ${ }^{44,63}$ Parasomniac episodes show various complex behaviors, often similar to wake behaviors ${ }^{83}$ (eg, rearranging furniture, inappropriate sexual activity, playing a musical instrument, driving a vehicle) ${ }^{6}$ Hence, these results suggest that the networks involved in BEp may be the same as those associated with motor planning during wakefulness.

Starting from the view that the episodes' complexity may express different cognitive processes underlying the BEp, we suggest that investigating EEG power dynamics and neural networks involved in association with the complexity of BEp may deserve interest.

Since most studies were limited to the central derivations ${ }^{20,34,43}$ or the midline, ${ }^{42,45}$ a critical issue remains the topographical distribution. As mentioned before, some inconsistent results could be affected by the limited number of derivations considered, such as the different dynamic evolution observed by Jaar et al and by Espa et al, or the different areas where SWA increases before the BEp. ${ }^{20,34,41-43,45}$

The few studies investigating cortical topography, assessing source localization and performing connectivity analysis, ${ }^{41,44,45}$ showed that also the theta activity was higher immediately before the BEp than during earlier time windows. ${ }^{45}$ Secondly, these studies observed an involvement of complex networks in the BEp, ${ }^{44,45}$ not restricted to central areas. Thus, we recommend future research to consider these analyses (ie, functional connectivity analysis and LORETA) and the use of high-density recordings to better define the network involved in the onset of BEp.

Lastly, the investigation of EEG patterns before BEp could be helpful in clinical practice. The NREM parasomnias can be associated with other medical conditions or sleep disorders (eg, obstructive sleep apneas, periodic leg movements, frontal lobe epilepsy) ${ }^{9,27,84}$ and the discrimination between these conditions could be difficult. Studies on the EEG topographical pattern suggest some differences in SWA between sleepwalking and other sleep 
disorders characterized by sleep fragmentation. ${ }^{85}$ Therefore, examining EEG patterns surrounding motor activations could shed light on both similar and different underlying processes, helping to better understand these sleep disorders.

This type of investigation seems to be promising in identifying processes involved in the onset of BEp. We recommend extending this investigation to other parasomnias, with the supplemental aim to clarify eventual differences in the mechanisms underlying these episodes.

\section{Conclusion}

Overall, the examined studies suggested that the electrophysiological features of SWS could play a key role in the onset of BEp. The SWA and SO increase in frontal and central areas might be an expression of the homeostatic sleep pressure, associated with higher sleep fragmentation. The presence of high-frequency EEG activity (ie, 24.00-$30.00 \mathrm{~Hz}$ ) immediately before the BEp was also shown. In view of this, the high- and low-frequency EEG increase before the BEp could represent a predictive EEG pattern of the BEp's onset.

The existence of different EEG activity patterns preceding the BEp could reflect eventual cognitive processes associated with these activations since some studies observed that a local increase of beta ${ }^{86,87}$ and gamma ${ }^{88}$ activity is associated with dream experience. Some authors recently proposed that the parasomniac episodes allow the unique opportunity to observe the mentation and eventual cognitive processes directly. ${ }^{89}$ Specifically, an "overt replay" of learning material during parasomnia activations was documented and these findings provide evidence for sleep-related learning as well as high concordance between the oneiric contents and the BEp. ${ }^{90-92}$ The investigation of EEG patterns before the BEp could represent a potential model to increase our knowledge about the mental processes involved in dreaming and memory consolidation. Furthermore, it might provide new insight into the study of cognitive processes during sleep.

In summary, the integration of different methodological protocols could fill the lack of consistent empirical findings, providing new insight into the study of NREM parasomnia pathophysiology.

\section{Disclosure}

The authors report no conflicts of interest in this work.

\section{References}

1. American Psychiatric Association. Diagnostic and Statistical Manual of Mental Disorders: DSM-5. American Psychiatric Association; 2013.

2. American Academy of Sleep Medicine. International Classification of Sleep Disorders: Diagnostic and Coding Manual. 3rd ed. 2014.

3. Sateia MJ. International Classification of Sleep Disorders-Third Edition. Chest. 2014;146(5):1387-1394. doi:10.1378/chest.14-0970

4. Rodrigues Brazete J, Montplaisir J, Petit D, et al. Electroencephalogram slowing in rapid eye movement sleep behavior disorder is associated with mild cognitive impairment. Sleep Med. 2013;14(11):1059-1063. doi:10.1016/j.sleep.2013.06.013

5. Fantini ML, Gagnon J-F, Petit D, et al. Slowing of electroencephalogram in rapid eye movement sleep behavior disorder. Ann Neurol. 2003;53(6):774-780. doi:10.1002/ana.10547

6. Montplasir J, Zadra A, Nielsen T, Petit D. Basic science, technical considerations and clinical aspects: fourth edition. Sleep Disord Med. 2017;1-1269.

7. Castelnovo A, Lopez R, Proserpio P, Nobili L, Dauvilliers Y. NREM sleep parasomnias as disorders of sleep-state dissociation. Nat Rev Neurol. 2018;14(8):470-481. doi:10.1038/s41582-018-0030-y

8. Fleetham JA, Fleming JAE. Parasomnias. CMAJ. 2014;186(8):E27380. doi: $10.1503 / \mathrm{cmaj} .120808$

9. Jacobson A, Kales A, Lehmann D, Zweizig JR. Somnambulism: all-night electroencephalographic studies. Science (80-). 1965;148 (3672):975-977. doi:10.1126/science.148.3672.975

10. Kavey NB, Whyte J, Resor SR Jr, Gidro-Frank S. Somnambulism in adults. Neurology. 1990;40(5):749-752. doi:10.1212/WNL.40.5.749

11. Zucconi M, Oldani A, Ferini-Strambi L, Smirne S. Arousal fluctuations in non-rapid eye movement parasomnias: the role of cyclic alternating pattern as a measure of sleep instability. J Clin Neurophysiol. 1995;12 (2):147-154. doi:10.1097/00004691-199503000-00005

12. Joncas S, Zadra A, Paquet J, Montplaisir J. The value of sleep deprivation as a diagnostic tool in adult sleepwalkers. Neurology. 2002;58(6):936-940. doi:10.1212/WNL.58.6.936

13. Zadra A, Desautels A, Petit D, Montplaisir J. Somnambulism: clinical aspects and pathophysiological hypotheses. Lancet Neurol. 2013;12(3):285-294. doi:10.1016/S1474-4422(12)70322-8

14. Blatt I, Peled R, Gadoth N, Lavie P. The value of sleep recording in evaluating somnambulism in young adults. Electroencephalogr Clin Neurophysiol. 1991;78(6):407-412. doi:10.1016/0013-4694(91) 90058-C

15. Gaudreau H, Joncas S, Zadra A, Montplaisir J. Dynamics of slow-wave activity during the NREM sleep of sleepwalkers and control subjects. Sleep. 2000;23(6):1-6. doi:10.1093/sleep/23.6.1d

16. Guilleminault C, Leger D, Philip P, Ohayon MM. Nocturnal wandering and violence: review of a sleep clinic population. J Forensic Sci. 1998;43(1):16103J. doi:10.1520/JFS16103J

17. Espa F, Dauvilliers Y, Ondze B, Billiard M, Besset A. Arousal reactions in sleepwalking and night terrors in adults: the role of respiratory events. Sleep. 2002;25(8):871-875. doi:10.1093/sleep/ 25.8.32

18. Lopez R, Jaussent I, Dauvilliers Y. Objective daytime sleepiness in patients with somnambulism or sleep terrors. Neurology. 2014;83 (22):2070-2076. doi:10.1212/WNL.0000000000001019

19. Lopez R, Shen Y, Chenini S, et al. Diagnostic criteria for disorders of arousal: a video-polysomnographic assessment. Ann Neurol. 2018;83 (2):341-351. doi:10.1002/ana.25153

20. Espa F, Ondze B, Deglise P, Billiard M, Besset A. Sleep architecture, slow wave activity, and sleep spindles in adult patients with sleepwalking and sleep terrors. Clin Neurophysiol. 2000;111(5):929-939. doi:10.1016/S1388-2457(00)00249-2

21. Guilleminault C, Kirisoglu C, da Rosa AC, Lopes C, Chan A. Sleepwalking, a disorder of NREM sleep instability. SLEEP Med. 2006;7(2):163-170. doi:10.1016/j.sleep.2005.12.006 
22. Castelnovo A, Riedner BA, Smith RF, Tononi G, Boly M, Benca RM. Scalp and source power topography in sleepwalking and sleep terrors: a high-density EEG Study. Sleep. 2016;39(10):1815-1825. doi: $10.5665 /$ sleep. 6162

23. Zucconi M, Ferri RB. Assessment of sleep disorders and diagnostic procedures. 1. Classification of sleep disorders. Eur Sleep Res Soc. 2014.

24. Parrino L, Ferri R, Bruni O, Terzano MG. Cyclic alternating pattern (CAP): the marker of sleep instability. Sleep Med Rev. 2012;16 (1):27-45. doi:10.1016/j.smrv.2011.02.003

25. Broughton R, Billings R, Cartwright R, et al. Homicidal somnambulism: a case report. Sleep. 1994

26. Crisp AH. The sleepwalking/night terrors syndrome in adults. Postgrad Med J. 1996;72(852):599-604. doi:10.1136/pgmj.72.852.599

27. Kales A, Jacobson A, Paulson MJ, Kales JD, Walter RD. Somnambulism: psychophysiological Correlates: i. All-Night EEG Studies. Arch Gen Psychiatry. 1966;14(6):586-594. doi:10.1001/ archpsyc.1966.01730120026004

28. Keefauver SP, Guilleminault C. Sleep terrors and sleepwalking. Princ Pract Sleep Med Philadelphia WB Saunders. 1994;567-573.

29. Crisp AH, Matthews BM, Oakey M, Crutchfield M. Sleepwalking, night terrors, and consciousness. Br Med J. 1990;300:360-362. doi:10.1136/bmj.300.6721.360

30. Halász P, Ujszászi J, Gádoros J. Are microarousals preceded by electroencephalographic slow wave synchronization precursors of confusional awakenings? Sleep. 1985;8(3):231-238. doi:10.1093/ sleep/8.3.231

31. Szelenberger W, Niemcewicz S, Dabrowska AJ. Sleepwalking and night terrors: psychopathological and psychophysiological correlates. Int Rev Psychiatry. 2005;17(4):263-270. doi:10.1080/ 09540260500104573

32. Pressman MR. Hypersynchronous delta sleep EEG activity and sudden arousals from slow-wave sleep in adults without a history of parasomnias: clinical and forensic implications. Sleep. 2004;27 (4):706-710. doi:10.1093/sleep/27.4.706

33. Guilleminault C, Moscovitch A, Leger D. Forensic sleep medicine nocturnal wandering and violence. Sleep. 1995;18(9):740-748. doi:10.1093/sleep/18.9.740

34. Guilleminault C, Poyares D, Aftab FA, Palombini L. Sleep and wakefulness in somnambulism: a spectral analysis study. $J$ Psychosom Res. 2001;51(2):411-416. doi:10.1016/S00223999(01)00187-8

35. Pilon M, Zadra A, Joncas S, Montplaisir J. Hypersynchronous delta waves and somnambulism: brain topography and effect of sleep deprivation. Sleep. 2006;29(1):77-84. doi:10.1093/sleep/29.1.77

36. Gibbs SA, Proserpio P, Terzaghi M, et al. Sleep-related epileptic behaviors and non-REM-related parasomnias: insights from stereo-EEG. Sleep Med Rev. 2016;25:4-20. doi:10.1016/j. smrv.2015.05.002

37. Moher D, Shamseer L, Clarke M, et al. Preferred reporting items for systematic review and meta-analysis protocols (PRISMA-P) 2015 statement. Rev Esp Nutr Humana y Diet. 2015.

38. Kwon Y, Lemieux M, McTavish J, Wathen N. Identifying and removing duplicate records from systematic review searches. J Med $\mathrm{Libr}$ Assoc. 2015;103(4):184-188. doi:10.3163/1536-5050.103.4.004

39. Wells G, Shea B, O'Connell D, et al. The Newcastle-Ottawa Scale (NOS) for assessing the quality of nonrandomised studies in meta-analyses. Ottawa, Ottawa Hosp Res Inst. 2020;2017.

40. Schenck CH, Pareja JA, Patterson AL, Mahowald MW. Analysis of polysomnographic events surrounding 252 slow-wave sleep arousals in thirty-eight adults with injurious sleepwalking and sleep terrors. $J$ Clin Neurophysiol. 1998;15(2):159-166. doi:10.1097/00004691199803000-00010

41. Zadra AL, Nielsen TA. Topographical EEG mapping in a case of recurrent sleep terrors. Dreaming. 1998;8(2):67-74. doi:10.1023/B: DREM.0000005897.62698.1b
42. Jaar O, Pilon M, Carrier J, Montplaisir J, Zadra A. Analysis of slow-wave activity and slow-wave oscillations prior to somnambulism. Sleep. 2010;33(11):1511-1516. doi:10.1093/sleep/ 33.11.1511

43. Perrault R, Carrier J, Desautels A, Montplaisir J, Zadra A Electroencephalographic slow waves prior to sleepwalking episodes. Sleep Med. 2014;15(12):1468-1472. doi:10.1016/j.sleep.2014.07.020

44. Januszko P, Niemcewicz S, Gajda T, et al. Sleepwalking episodes are preceded by arousal-related activation in the cingulate motor area: EEG current density imaging. Clin Neurophysiol. 2016;127 (1):530-536. doi:10.1016/j.clinph.2015.01.014

45. Desjardins M, Carrier J, Lina J, et al. EEG Functional Connectivity Prior to Sleepwalking: evidence of Interplay Between Sleep and Wakefulness. Sleep. 2017;40(4). doi:10.1093/sleep/zsx024.

46. Rechtschaffen A, Kales A. A manual of standardized terminology, techniques and scoring system for sleep stages of human subjects. UCLA Brain Inf Serv Los Angeles. 1978.

47. Mölle M, Marshall L, Gais S, Born J. Grouping of spindle activity during slow oscillations in human non-rapid eye movement sleep. J Neurosci. 2002;22(24):10941-10947. doi:10.1523/ JNEUROSCI.22-24-10941.2002

48. Dang-Vu TT, Schabus M, Desseilles M, et al. Spontaneous neural activity during human slow wave sleep. Proc Natl Acad Sci US A. 2008;105(39):15160-15165. doi:10.1073/pnas.0801819105

49. Massimini M, Huber R, Ferrarelli F, Hill S, Tononi G. The sleep slow oscillation as a traveling wave. J Neurosci. 2004;24:6862-6870. doi:10.1523/JNEUROSCI.1318-04.2004

50. Carrier J, Viens I, Poirier G, et al. Sleep slow wave changes during the middle years of life. Eur $J$ Neurosci. 2011;33(4):758-766. doi:10.1111/j.1460-9568.2010.07543.x

51. Bonnet M, Carley D, Consultant MC, et al. The Atlas Task Force: EEG arousals: scoring rules and examples. Sleep. Sleep Rochester. 1992.

52. Silber MH, Ancoli-Israel S, Bonnet MH, et al. The visual scoring of sleep in adults. J Clin Sleep Med. 2007;03(02):121-131. doi:10.5664/ jcsm. 26814

53. Lopez R, Jaussent I, Scholz S, Bayard S, Montplaisir J, Dauvilliers Y. Functional Impairment in Adult Sleepwalkers: a Case-Control Study. Sleep. 2013;36(3):345-351. doi:10.5665/sleep. 2446

54. Steriade M. Grouping of brain rhythms in corticothalamic systems. Neuroscience. $\quad 2006 ; 137(4): 1087-1106 . \quad$ doi:10.1016/j. neuroscience.2005.10.029

55. Bassetti C, Vella S, Donati F, Wielepp P, Weder B. SPECT during sleepwalking. Lancet. 2000;356(9228):484-485. doi:10.1016/S01406736(00)02561-7

56. Steriade M, McCarley RW. Brainstem control of wakefulness and sleep; 1990.

57. Pilon M, Zadra A, Joncas S, Rompré S, Montplaisir J. Hypersynchronous delta activity and somnambulism: differences between frontal and central leads. Sleep. 2003;26.

58. Pilon M, Montplaisir J, Zadra A. Precipitating factors of somnambulism symbol: impact of sleep deprivation and forced arousals. Neurology. 2008;70:2284-2290. doi:10.1212/01.wnl.000030408 2.49839 .86

59. Zadra A, Pilon M, Montplaisir J. Polysomnographic diagnosis of sleepwalking: effects of sleep deprivation. Ann Neurol. 2008;63 (4):513-519. doi:10.1002/ana.21339

60. Broughton R. NREM arousal parasomnias. In: Kryger MC, Roth T, Dement WC, editors. Principles and Practice of Sleep Medicine. 3rd ed. Philadelphia: W.B. Saunders; 2000:693-706.

61. Pressman MR. Factors that predispose, prime and precipitate NREM parasomnias in adults: clinical and forensic implications. Sleep Med Rev. 2007;11(1):3-5. doi:10.1016/j.smrv.2006.06.003

62. Hoffstaedter F, Grefkes C, Zilles K, Eickhoff SB. The "what" and "when" of self-initiated movements. Cereb Cortex. 2013;23 (3):520-530. doi:10.1093/cercor/bhr391 
63. Kremer S, Chassagnon S, Hoffmann D, Benabid AL, Kahane P. The cingulate hidden hand [1]. J Neurol Neurosurg Psychiatry. 2001;70:264-265. doi:10.1136/jnnp.70.2.264

64. Talairach J, Bancaud J, Geier S, et al. The cingulate gyrus and human behaviour. Electroencephalogr Clin Neurophysiol. 1973;34(1):45-52. doi:10.1016/0013-4694(73)90149-1

65. Terzaghi M, Sartori I, Tassi L, et al. Evidence of dissociated arousal states during NREM parasomnia from an intracerebral neurophysiological Study. Sleep. 2009;32(3):409-412. doi:10.1093/sleep/ 32.3.409

66. Terzaghi M, Sartori I, Tassi L, et al. Dissociated local arousal states underlying essential clinical features of non-rapid eye movement arousal parasomnia: an intracerebral stereo-electroencephalographic study. J Sleep Res. 2012;21(5):502-506. doi:10.1111/j.13652869.2012.01003.x

67. Flamand M, Boudet S, Lopes R, et al. Confusional arousals during non-rapid eye movement sleep: evidence from intracerebral recordings. Sleep. 2018;41(10). doi:10.1093/sleep/zsy139.

68. Siclari F, Tononi G. Local aspects of sleep and wakefulness. Curr Opin Neurobiol. 2017;44:222-227. doi:10.1016/j.conb.2017.05.008

69. Stallman HM, Kohler M. Prevalence of sleepwalking: a systematic review and meta-analysis. PLoS One. 2016;11(11):e0164769. doi:10.1371/journal.pone.0164769

70. Ohayon MM, Mahowald MW, Dauvilliers Y, Krystal AD, Léger D. Prevalence and comorbidity of nocturnal wandering in the US adult general population. Neurology. 2012;78(20):1583-1589. doi:10.1212/ WNL.0b013e3182563be5

71. Laberge L, Tremblay RE, Vitaro F, Montplaisir J. Development of parasomnias childhood to early adolescence. Pediatrics. 2000;106 (1I):67-74. doi:10.1542/peds.106.1.67

72. Gorgoni M, D’Atri A, Scarpelli S, Reda F, De Gennaro L. Sleep electroencephalography and brain maturation: developmental trajectories and the relation with cognitive functioning. Sleep Med. 2020;66:33-50. doi:10.1016/j.sleep.2019.06.025

73. Scarpelli S, Bartolacci C, D’Atri A, Gorgoni M, De Gennaro L. Mental sleep activity and disturbing dreams in the lifespan. Int J Environ Res Public Health. 2019;16(19):3658. doi:10.3390/ ijerph16193658

74. Carpentier N, O'Reilly C, Carrier J, et al. Spindles insufficiency in sleepwalkers' deep sleep. Neurophysiol Clin. 2020;50(5):339-343. doi:10.1016/j.neucli.2020.08.003

75. Sarilar AC, Ismailogullari S, Yilmaz R, Erdogan FF, Per $H$. Electroencephalogram abnormalities in patients with NREM parasomnias. Sleep Med. 2019;3-7.

76. Wauquier A, Aloe L, Declerck A. K-complexes: are they signs of arousal or sleep protective? J Sleep Res. 1995;4(3):138-143. doi:10.1111/j.1365-2869.1995.tb00162.x

77. Nicholas CL, Trinder J, Colrain IM. Increased production of evoked and spontaneous K-complexes following a night of fragmented sleep. Sleep. 2002;25(8):42-47. doi:10.1093/sleep/25.8.42

78. Gorgoni M, Reda F, D’Atri A, Scarpelli S, Ferrara M, De Gennaro L. The heritability of the human K-complex: a twin study. Sleep. 2019;42(6). doi:10.1093/sleep/zsz053

Nature and Science of Sleep

\section{Publish your work in this journal}

Nature and Science of Sleep is an international, peer-reviewed, open access journal covering all aspects of sleep science and sleep medicine, including the neurophysiology and functions of sleep, the genetics of sleep, sleep and society, biological rhythms, dreaming, sleep disorders and therapy, and strategies to optimize healthy sleep.
79. De Gennaro L, Ferrara M, Bertini M. Topographical distribution of spindles: variations between and within NREM sleep cycles. Sleep Res Online. 2000;3(4):155-160.

80. Curcio G, Ferrara M, Pellicciari MC, Cristiani R, De Gennaro L. Effect of total sleep deprivation on the landmarks of stage 2 sleep. Clin Neurophysiol. 2003;114(12):2279-2285. doi:10.1016/S13882457(03)00276-1

81. Lecendreux M, Bassetti C, Dauvilliers Y, Mayer G, Neidhart E, Tafti M. HLA and genetic susceptibility to sleepwalking. Mol Psychiatry. 2003;8(1):114-117. doi:10.1038/sj.mp.4001203

82. Marzano C, Ferrara M, Curcio G, De Gennaro L. The effects of sleep deprivation in humans: topographical electroencephalogram changes in non-rapid eye movement (NREM) sleep versus REM sleep. J Sleep Res. 2010.

83. Arnulf I, Uguccioni G, Gay F, et al. What does the sleeping brain say? Syntax and semantics of sleep talking in healthy subjects and in parasomnia patients. Sleep. 2017;40(11). doi:10.1093/sleep/zsx159.

84. Foldvary-Schaefer N, Alsheikhtaha Z. Complex nocturnal behaviors: nocturnal seizures and parasomnias. Continuum (Minneap Minn). 2013;19(1Sleep Disorders):104-131. doi:10.1212/01. CON.0000427210.98305.8f

85. Jones SG, Riedner BA, Smith RF. Regional reductions in sleep electroencephalography power in obstructive sleep apnea: a high-density EEG Study. Sleep. 2014.

86. Scarpelli S, D'atri A, Bartolacci C, et al. Dream recall upon awakening from non-rapid eye movement sleep in older adults: electrophysiological pattern and qualitative features. Brain Sci. 2020;10:343. doi:10.3390/brainsci10060343

87. Scarpelli S, Bartolacci C, D'Atri A. et al. Electrophysiological correlates of dream recall during REM Sleep: evidence from multiple awakenings and within-subjects design. Nat Sci Sleep;2020. 1043-1052. doi:10.2147/NSS.S279786

88. Siclari F, Baird B, Perogamvros L, et al. The neural correlates of dreaming. Nat Neurosci. 2017;20(6):872-878. doi:10.1038/nn.4545

89. Alfonsi V, D'Atri A, Scarpelli S, Mangiaruga A, De Gennaro L. Sleep talking: a viable access to mental processes during sleep. Sleep Med Rev. 2019;44:12-22. doi:10.1016/j.smrv.2018.12.001

90. Limousin N, Dehais C, Gout O, Heran F, Oudiette D, Arnulf I. A brainstem inflammatory lesion causing REM sleep behavior disorder and sleepwalking (parasomnia overlap disorder). Sleep Med. 2009;10(9):1059-1062. doi:10.1016/j.sleep.2008.12.006

91. Uguccioni G, Pallanca O, Golmard J, Dodet P, Herlin B. Sleeprelated declarative memory consolidation and verbal replay during sleep talking in patients with REM sleep behavior disorder. PLoS One. 2013;8(12):4-11. doi:10.1371/journal.pone.0083352

92. Oudiette D, Constantinescu I, Leclair-Visonneau L, et al. Evidence for the re-enactment of a recently learned behavior during sleepwalking. PLoS One. 2011;6(3):e18056. doi:10.1371/journal. pone. 0018056
The manuscript management system is completely online and includes a very quick and fair peer-review system, which is all easy to use. Visit http://www.dovepress.com/testimonials.php to read real quotes from published authors. 